\title{
Solutions for Porte's Conjectures
}

\author{
By
}

\section{Michiro KONDO*}

J. Porte have provided a series of very weak modal logics in [1]. First of all he introduced the weakest modal logic Sa in that series and defined the series by applying the operations "6the reinforcement" and "the normalization" to Sa repeatedly. The well-known modal logics (e. g. T, S4, etc.) are contained in this series. Unfortunately, since these logics are rather weak, many problems (e.g.the completeness theorems by means of Kripke's semantics) remain open. In [2], Porte has left the following conjectures.

Conjecture (III): $\rho \nu S a$ is complete for the model structure $\langle G, K, R\rangle$ where the real world is normal, while all the other worlds are seminormal (or normal), the relation $R$ being reflexive and transitive.

Conjecture $(\mathbb{I V})$ : If the model structure is like in conjecture (III), except that $R$ is not bound to be transitive, it determines a logic which can be axiomatized by $\nu P 1, \nu P 2, \nu P 3, D, \rho \nu D, \rho W, I$.

But, judging from the context, probably the conjecture (IV) is to be read as follows.

Conjecture $\left(\mathbb{H} \mathbb{V}^{*}\right)$ : If the model structure is like in conjecture (III), except that $R$ is bound to be transitive, it determines a logic which can be axiomatized by $\nu P 1, \nu P 2, \nu P 3, D, \rho \nu D, \rho W, I$.

In this paper, I will give the affirmative solution for (III) and negative one for $\left(\mathbb{I V}^{*}\right)$.

Communicated by S. Takasu, May 24, 1986. Revised March 2, 1987

* Department of Mathematics, Rikkyo University, Tokyo 171, Japan 


\section{§1. The Logic $\mathbb{H}$ and Its Semantics}

For the sake of simplicity we denote the logics in (III) and (IV*) as $H$ and $H^{\wedge}$ respectively. First of all we shall state the logic $H$.

The axiom system of $H$ is following.

Axiom schemata:

$$
\begin{array}{lll}
A 1 & (P 1) & A \rightarrow(B \rightarrow A) \\
A 2 & (P 2) & (A \rightarrow(B \rightarrow C)) \rightarrow((A \rightarrow B) \rightarrow(A \rightarrow C)) \\
A 3 & (P 3) & (\sim A \rightarrow \sim B) \rightarrow(B \rightarrow A) \\
A 4 & (\nu P 1) & L(A \rightarrow(B \rightarrow A)) \\
A 5 & (\nu P 2) & L((A \rightarrow(B \rightarrow C)) \rightarrow((A \rightarrow B) \rightarrow(A \rightarrow C))) \\
A 6 & (\nu P 3) & L((\sim A \rightarrow \sim B) \rightarrow(B \rightarrow A)) \\
A 7 & (\rho D) & L(A \rightarrow B) \rightarrow(L A \rightarrow L B) \\
A 8 & (\rho W) & L A \rightarrow A \\
A 9 & (\rho I) & L A \rightarrow L L A
\end{array}
$$

Rule of inference:

$R 1(D)$ infer $B$ from $A \rightarrow B$ and $A$, where we use the symbols $A 1, \ldots, A 9$ and $R 1$ instead of Porte's notation $(P 1), \ldots,(\rho I)$ and $(D)$, respectively.

Hence the logic $H$ is obtained from SO. 5 by adding an extra axiom scheme $A 9 L A \rightarrow L L A$ to the axiom system of SO.5.

A structure $M=\langle G, K, R, V\rangle$ is called an $H$-model (or simply model if no confusion arises) if $G \in K, R$ is a reflexive and transitive relation on $K$, and $V$ is the valuation function satisfying the conditions: For every $u \in K$ and any formulas $A$ and $B$ of $H$,

(C1) $\quad V(\sim A, u)=1 \quad$ iff $\quad V(A, u)=0$

(C2) $\quad V(A \rightarrow B, u)=1 \quad$ iff $\quad V(A, u)=0$ or $V(B, u)=1$

(C3) $V(L A, G)=1$ iff $V(A, u)=1$ for every $u$ such that GRu

(C4) if $V(L A, u)=0$, then $V(A, v)=0$ for some $v$ such that $u R v$.

A semi-normal world $w$ is one satisfying the following condition: For every formula $L A$, if $V(L A, w)=0$, then there exists $w^{\prime} \in K$ accessible to $w$ (i. e. $\left.w R w^{\prime}\right)$ such that $V\left(A, w^{\prime}\right)=0$.

A normal world $w$ is one satisfying the condition: For every formula $L A, V(L A, w)=1$ if and only if $V\left(A, w^{\prime}\right)=1$ for every $w^{\prime}$ such that $w R w^{\prime}$. 
Hence $G$ is normal while all the other worlds are semi-normal in any $H$-model.

A formula $A$ of $H$ is said to be true in an $H$-model $M=\langle G, K$, $R, V\rangle$ when $V(A, G)=1$. A formula $A$ of $H$ is said to be $H$-valid (simply valid unless confusion arises) if $A$ is true in every $H$-model.

Thus (III) means that $A$ is provable in $H(H \vdash A)$ iff $A$ is $H$-valid. Therefore, the conjecture (III) follows from the completeness theorem of $H$ in terms of the models stated above.

First, we show the soundness theorem of $H$.

\section{Theorem 1. If $H \vdash A$, then $A$ is $H$-valid.}

Proof. It is sufficient to show that every axiom scheme is valid and the rule of inference preserves validity. We show only the cases of $A 8$ and $A 9$.

For the case of $A 8$, suppose that $A 8$ is not valid. There exists a model $\langle G, K, R, V\rangle$ such that $V(A 8, G)=0$. That is, $V(L A, G)=1$ but $V(A, G)=0$. Since $R$ is reflexive, we have $G R G$. Hence we obtain $V(A, G)=1$ by $V(L A, G)=1$. This is a contradiction.

Suppose that $A 9$ is not valid. There is a model $\langle G, K, R, V\rangle$ such that $V(L A, G)=1$ but $V(L L A, G)=0$. Since $V(L L A, G)=0$, we get $V(L A, u)=0$ for some $u$ such that $G R u$. Since $u$ is semi-normal, $V(A, v)=0$ for some $v$ such that $u R v$. By the transitivity of $R$, we obtain $G R v$. Hence we have $V(A, v)=1$ by $V(L A, G)=1$. But this is a contradiction.

Therefore the soundness theorem of $H$ can be proved.

\section{§2. Completeness of $\mathbb{H}$}

We shall establish the completeness theorem of $H$. We need some definitions and lemmata in order to show the theorem.

First we define a logic $S L$. The language of a logic $S L$ is as follows: propositional variables $p, q, r, \ldots$; primitive connectives $\sim, \rightarrow$ and $L$. The logic $S L$ has the axiom schemata $A 1-A 3$ and the rule of inference $R 1$. Thus, roughly speaking, $S L$ has the same structure as the classical propositional logic. But $S L$ has the formulas of the form $L A$. 
A set $S$ of formulas of a given logic $X$ is called consistent in $X$ if there are no formulas $A_{1}, \ldots, A_{n}$ of $S$ such that $X \vdash \sim\left(A_{1} \wedge \ldots \wedge A_{n}\right)$. Otherwise $S$ is called inconsistent in $X$.

A consistent set $S$ in $X$ is said to be maximal consistent in it if $A \in S$ or $\sim A \in S$ for every formula $A$ of $X$.

Simple calculation yields the next facts: For every maximal consistent set $S$ in $X$,

(1) if $A$ is provable in $X$, then $A \in S$

(2) if $A \in S$ and $A \rightarrow B \in S$, then $B \in S$.

As to the logic $S L$, we have the next theorem.

Theorem 2. If $S L \vdash A$, then $H \vdash L A$.

Proof. We can prove easily this theorem by induction on the proof of $A$ in $S L$.

We shall state the useful lemma without proof.

Lemma 3. (Lindenbaum's lemma) If $S$ is a consistent set in $X$, then there exists a maximal consistent set $U$ in $X$ containing $S$.

And we note also the following lemma.

Lemma 4. If $S$ is a maximal consistent set in $H$ and $L A \oplus S$, then there is a maximal consistent set $U$ in $S L$ such that $\sim A \in U$ and $B \in U$ for every formula $B$ satisfying $L B \in S$.

\section{Proof. Immediate from Lemma 3 and Routley [4] Lemma 4.}

We establish the completeness theorem of $H$. Suppose that $A$ is not provable in $H$. We shall show that $A$ is not valid in our semantics. Since $\{\sim A\}$ is consistent in $H$, there is a maximal consistent set $G$ in $H$ containing $\{\sim A\}$ by Lemma 3 .

Let $K$ be the set of all maximal consistent sets $u$ in $S L$ such that $(\forall B) \quad(L B \in G \Rightarrow B \in u)$. Clearly $G \in K$. We define the relation $R$ as follows: For every $u, v \in K, u R v$. Clearly $R$ is a reflexive and transitive relation on $K$. Lastly we define the valuation function $V$. Let 
$V$ be the function satisfying the condition: For every formula $B$ and every element $u \in K, V(B, u)=1$ iff $B \in u$.

Now, we have to check that $V$ is the valuation function in our sense. For $(C 1)$ and $(C 2)$, it is trivial。 For (C3), suppose that $V(L B, G)=1$. Since $L B \in G$, we have that $B \in u$ and thus $V(B, u)=1$ for every $u$ such that $G R u$. Conversely, suppose that $V(L B, G)=0$ 。 Since $L B \notin G$ and $G$ is a maximal consistent set in $H$, by Lemma 4 , there is a maximal consistent set $u$ in $S L$ such that $\sim B \in u$. As to this $u$, we have $u \in K$ and $G R u$. These facts mean that if $V(L B, G)=0$ then $V(B, u)=0$ for some $u$ such that $G R u$. And as to (C4), suppose that $V(L B, u)=0$. Since $L B \notin u$, we have $L L B \notin G$ by definition of $K$. Since $L B \rightarrow L L B$ is provable in $H$, we have $L B \rightarrow L L B \in G$ and hence $L B \notin G$. Thus there exists a maximal consistent set $v \in K$ such that $\sim B \in v$ 。 We have $u R v$. Thus if $V(L B, u)=0$, then $V(B, v)=0$ for some $v$ such that $u R v$.

Now we are going to show the completeness theorem of $H$. Since $H \not H A$ and $\sim A \in G$, we have $V(A, G)=0$ for the model $\langle G, K, R, V\rangle$ constructed above. This means that

Theorem 5. If $A$ is $H$-valid, then $H \vdash A$.

This indicates that the conjecture (III) holds.

Checking the above argument, we have another result for (III).

Let $\langle G, K, R, V\rangle$ be a structure such that $G \in K, R$ is a restricted reflexive relation on $K$ (i. e. $G R G$ ) and a restricted transitive one (i. e. if $G R u$ and $u R v$ then $G R v$ ), and $V$ is a valuation function satisfying $(C 1)-(C 4)$. We call this structure an $H^{*}$-model.

Then we can prove the next result without difficulty.

Theorem 6. The conjecture (III) holds for $H^{*}$-model structures.

\section{§ 3. A Solution for the Conjecture $\left(\mathbb{I V}^{*}\right)$}

The logic $H^{\wedge}$ in the conjecture $\left(\mathrm{IV}^{*}\right)$ has the axiom schemata $A 1-A 8$ and the rules of inference $R 1$ and $R 2$ (I); infer $L L A$ from $L A$. Hence $H^{\wedge}$ is obtained from $\mathrm{SO} .5$ by adding the extra rule of inference $R 2$ to the axiom system to it. 
We call a structure $\langle G, K, R, V\rangle$ an $H^{\wedge}$-model (simply model) if a normal world $G \in K, R$ is a reflexive relation on $K$, and $V$ is a valuation function satisfying $(C 1)-(C 4)$ in $\S 1$.

An $H^{\wedge}$-formula $A$ is said to be true in an $H^{\wedge}$-model $\langle G, K, R, V\rangle$ when $V(A, G)=1$. A formula $A$ is called $H^{\wedge}$-valid (simply valid) if for every $H^{\wedge}$-model $\langle G, K, R, V\rangle$ we have $V(A, G)=1$. Therefore the conjecture (IV*) means that $H^{\wedge} \vdash A$ iff $A$ is valid.

Unfortunately the conjecture does not hold. Because, since $R 2$ does not preserve validity, even the soundness theorem does not hold. Suppose that $L A$ is valid but $L L A$ is not. There is a model $\langle G, K$, $R, V>$ such that $V(L L A, G)=0$. Thus $V(L A, u)=0$ for some $u$ with $G R u$. However $u$ is not necessarily a normal world and therefore our assumption does not necessarily lead to a contradiction. Thus the conjecture $\left(\mathrm{IV}^{*}\right)$ does not hold.

However, we can get the completeness theorem for $H^{\wedge}$ if we modify the definition of $H^{\wedge}$-models slightly as mentioned below.

We call a structure $\langle N, K, R, V\rangle$ an $H^{\wedge} *$-model (simply $*$-model) when $N$ is a non-empty subset of $K$ and $R$ is a reflexive relation on $K$ with the extra condition; for $u, v \in K, u R v$ implies $x R v$ for some $x \in N$. And $V$ is a valuation function satisfying the conditions $(C 1)$, $(C 2),(C 4)$ and the following condition $\left(C 3^{*}\right)$.

$\left(C 3^{*}\right)$ For $u \in N, V(L A, u)=1$ iff $V(A, v)=1$ for every $v$ with $u R v$.

Hence every world in $N$ is normal while all the other worlds are semi-normal in any $*$-model.

An $H^{\wedge}$-formula $A$ is valid when, for every $H^{\wedge} *$-model $\langle N, K, R$, $V>$, we have $V(A, u)=1$ for every $u \in N$.

Now we shall show the soundness theorem for $H^{\wedge}$.

Theorem 7. If $H^{\wedge} \vdash A$, then $A$ is valid.

Proof. We show only that the rule $R 2$ preserves validity. Suppose that $L A$ is valid but $L L A$ is not. There is a $*$-model $\langle N, K, R, V\rangle$ such that $V(L L A, u)=0$ for some $u \in N$. We have $V(L A, v)=0$ for some $v$ with $u R v$. By $(C 4)$, there is $w$ such that $v R w$ and $V(A, w)=0$. By $v R w$ and the condition of $R$, we have $x R w$ for some $x \in N$. Since $L A$ is valid, we have $V(L A, x)=1$ and hence $V(A, w)=1$. But this is a contradiction. Thus $R 2$ preserves validity. 
Next we shall prove the completeness theorem.

Theorem 8. If $A$ is valid, then $H^{\wedge} \vdash A$.

Proof. Suppose that $H^{\wedge} \forall A$. It is sufficient to prove that $A$ is not valid. Since $H^{\wedge} \forall A,\{\sim A\}$ is consistent in $H^{\wedge}$. Hence there is a maximal consistent set $G$ in $H^{\wedge}$ containing $\{\sim A\}$. Now, let $N$ be the set of all maximal consistent sets in $H^{\wedge}$. Let $K$ be the set of all maximal consistent sets $u$ in $S L$ such that $(\exists x \in N)(\forall B)(L B \in x \Rightarrow$ $B \in u)$. It is easy to show that $N$ is a non-empty subset of $K$. Next we define the relation $R$ on $K$ as follows: For $u, v \in K, u R v$ iff (i) $u \in N$ and $(\forall B) \quad(L B \in u \Rightarrow B \in v)$ or (ii) $u \notin N$ and $(\exists y \in N) \quad(\forall B)$ $(L B \in y \Rightarrow B \in v)$. Lastly we define $V$ as in $\S 2$.

We have to check that this structure $\langle N, K, R, V\rangle$ is a $*$-model. We show only that $V$ satisfies $(C 4)$. Suppose that $V(L B, u)=0$. If $u \in N$, by Lemma 4, then $V$ clearly satisfies (C4). So we suppose that $u \notin N$. By definition of $K$, there is $x \in N$ such that $C \in u$ for every formula $C$ satisfying $L C \in x$. Hence we have $L L B \notin x$ by $L B \notin u$. This means that $H^{\wedge} \forall L L B$. By $R 2$ we get $H^{\wedge} \forall L B$. Hence there is a maximal consistent set $y$ in $H^{\wedge}$ containing $\{\sim L B\}$. Since Lemma 4 holds also for $H^{\wedge}$, there is a maximal consistent set $v$ in $K$ such that $B \notin v$ and $C \in v$ for every formula $C$ satisfying $L C \in y$. Thus, $v$ is also in $K$. By definitions of $R$ and $V$, we have that $V(B, v)=0$ for some $v$ with $u R v$.

Thus the structure $\langle N, K, R, V\rangle$ is a $*$-model. Since $\sim A \in G \in N$, we have that $V(A, G)=0$ for some $G \in N$. This means that $A$ is not valid. Hence the completeness theorem for $H^{\wedge}$ can be proved.

\section{Acknowledgement}

I wish to thank referee for the valuable suggestions especially for $\S 2$. Moreover he showed me that the conjecture (III) holds for the model structures $(G, K, R, V\rangle$ such that $R$ is an equivalence relation an $K$.

\section{References}

[1] Porte, J., A research in modal logic, Logique et Analy., 23 (1980), 3-34. 
[2] Notes on modal logic, Logique et Analy. (N. S.), 24 (1981), 396-406.

[3] Cresswell, M. J., The completeness of SO. 5, Logique et Analy., 9 (1966), 263-266.

[4] Routley, R., Extensions of Makinson's completeness theorems in modal logic, Zeitschr. f. math. Logik und Grundl. d. Math., 16 (1970), 239-256. 